enkephalin offers the long sought approach to the development of a nonaddictive analgesic. Kosterlitz and Hughes (Life Sci., 17, 91; 1975) have described some preliminary evidence in favour of this view. They point out that enkephalin has some unusual pharmacological properties resembling those seen in certain recently developed synthetic drugs of the benzomorphan class, which seem to be free of addictive properties in monkey tests. These properties are, first, a relatively rapid rate of onset and short duration for the effects on opiate receptors in isolated test organs and second that the actions are less readily blocked by standard antagonist drugs such as naloxone. To antagonise a response of given magnitude requires about a four times higher concentration of naloxone when enkephalin is the agonist than when conventional agonists such as normorphine are used.

There will also undoubtedly be rapid progress in establishing radioimmunoassay methods to measure enkephalin in brain, and possibly immunohistochemical techniques to elucidate its precise cellular localisation in different brain regions. This information should help to clarify the possible physiological role of enkephalin as a neurotransmitter or neuromodulator substance associated with pain pathways in brain.

\title{
A photosynthetic structure
}

\section{from A. G. Lee}

READING the literature on photosynthesis is almost certainly bad for the health. To be told that in the green photosynthetic bacteria, exposure to light causes a flow of electrons from Chl-660 to Chl-770 then to P840 and finally to C551, C553 and C555 does little to reassure one, especially when informed that Chl-770 is also called B810, that P840 could be an aggregated form of $\mathrm{Chl}-770$ and that C551 and C555 also masquerade under the names C419 and C422 respectively. These names of course reflect the fact that the physical and chemical identity of the species are generally unknown, and that they have been recognised only from changes in absorption spectra. For any satisfactory model of photosynthesis, it will be necessary to translate these labels into actual chemical species present within the photosynthetic system. In a paper by Fenna and Matthews in this issue of Nature, (page 573), a start is made towards this goal.

In the green bacterium Chlorobium limicola (once called Chloropseudomonas ethylica) the photosynthetic apparatus is contained within egg. shaped vesicles, about $50 \mathrm{~nm}$ wide and $150 \mathrm{~nm}$ long, arranged as a layer along the inside face of the cytoplasmic membrane. In this it differs from the purple photosynthetic bacteria, where the photosynthetic membrane is continuous with the cytoplasmic membrane. The photosynthetic apparatus in the green bacteria also differs in that they contain an extrinsic chlorophyllcontaining protein which is water soluble and can be removed by changes in buffer strength and $p \mathrm{H}$ : the chlorophyll-containing proteins in purple bacteria and plants are firmly attached to membranes and can only be removed by detergent treatment. Because it is water soluble, it has been possible to purify and crystallise this chlorophyllprotein from $C$. limicola, and it is the structure of this protein that is reported by Fenna and Matthews.

Although the bulk of the chlorophyll in $C$. limicola is chlorobium chlorophyll (also called bacterioviridin or Chl-660), there is present as a minor component bacteriochlorophyll (Chl-770, also called, you will remember, $\mathrm{B} 810$ ), and it is bacteriochlorophyll that is contained in the water-soluble protein. Since the amino-acid sequence of the protein is unknown, the X-ray crystallographic studies only give information about the $\alpha$-carbon backbone, but the structure is sufficiently defined to show the basic protein conformation.

The protein consists of three identical subunits, each containing in its centre seven bacteriochlorophyll molecules arranged in an apparently irregular fashion. The structure of each subunit is described as a distorted hollow cylinder, with one end of the cylinder possibly being open. The interesting and unique aspect of the structure is the hollow cavity within each cylindrical subunit, with the bacteriochlorophyll molecules being bound to the 'walls' of this cavity. The binding of the bacteriochlorophyll molecules to the protein is partly due to interactions between the phytyl chains and what are probably hydrophobic residues facing towards the centre of the subunit. There are also, however, extensive interactions involving the $\mathrm{Mg}$ ions of the porphine rings, either through hydrogen bonding between the protein and a water molecule coordinated to the $\mathrm{Mg}$, or through a direct interaction between the $\mathrm{Mg}$ and a suitable coordinating group of the peptide. Hydrogen bonding is also possible between some of the substituents of the bacteriochlorophyll and the protein. Although there seems to be no direct contact between the porphine rings, the average centre-to-centre nearest neighbour distance is only $12 \mathrm{~A}$, so that efficient energy transfer is possible within each subunit.

If the structure of this particular chlorophyll-containing protein is at all typical, then it makes the derivation of molecular models from absorption spectra seem an almost impossible task, since although the planes of the seven porphine rings in each subunit are close to being parallel, there is no obvious pattern to their relative orientation. The question as to how important are the chlorophyll-proteins generally in the photosynthetic process still remains open however. The actual role of the bacteriochlorophyll-protein for example is still uncertain : although it has been suggested that it acts to transfer excitation from the antenna chlorobium Chl-660 to the reaction centre P840, it has not yet been demonstrated that photosynthesis fails to occur in its absence. The fact that it is water soluble also makes it highly atypical. The isolation of chlorophyllcontaining proteins from other photosynthetic systems requires the use of detergents, and it is not then at all clear whether the isolated complexes correspond to species actually present in the membrane. Thus although membrane-bound proteins are generally isolated in detergent solutions as lipidprotein complexes, this does not mean that they were present in that form in the membrane: the presence of an organised lipid bilayer in the membrane means that hydrophobic surfaces of the membrane proteins are isolated from the aqueous phase, whereas in the detergent solution, a specific lipidprotein interaction is necessary to provide the same protection. The observation that the majority of the chlorophyll in a photosynthetic membrane is found associated with protein after detergent treatment similarly does not mean that it was the same in the membrane. In fact, it is known that chlorophyll incorporated into a lipid bilayer in the absence of any protein will adopt an aggregated structure analogous to that proposed for antenna chlorophyll (Strouse, Proc. natn. Acad. Sci. U.S.A., 71, 325-328; 1971). Nevertheless, it is obvious that chlorophyll in the reaction centres must be closely associated with protein, and the structure reported by Fenna and Matthews shows something of the complexity that might be expected. 